\title{
La sede Valparaíso de la Universidad de Chile (1968-1981): Preludio de la Universidad de Valparaíso
}

The Campus Valparaíso of the University of Chile (1968-1981): Prelude of the University of Valparaíso

\author{
Manuel Patricio Vergara Rojas \\ Universidad de las Américas, Chile \\ mvergararojas@gmail.com
}

\section{Resumen}

La creación de la Sede Valparaíso de la Universidad de Chile, después de varios años de presencia de sus escuelas e institutos en la provincia de Valparaíso, es un evento relevante en el proceso de reforma universitaria de la Casa de Bello y, también, en la historia de la educación superior de la ciudad puerto. El quiebre institucional de 1973 tuvo como consecuencia un segundo proceso que determinó la creación de las dos actuales universidades estatales de la región: la Universidad de Valparaíso y, al poco tiempo después, la Universidad de Playa Ancha, lo que, por una parte, otorgó una anhelada autonomía a las universidades regionales, pero desperdició la oportunidad de establecer un moderno sistema universitario estatal.

Palabras claves: Universidad de Chile; Reforma Universitaria; Sede Valparaíso; Contrarreforma Universitaria; Universidad de Valparaíso.

\begin{abstract}
The creation of the Campus Valparaíso of the University of Chile, after several years of presence of its schools and institutes in the province of Valparaíso, is an important event in the process of university reform of the House of Bello and, also, in the history of higher education of the port city. The institutional breakdown of 1973 resulted in a second process that determined the creation of the two current state universities in the region: the University of Valparaíso and, shortly thereafter, the University of Playa Ancha, which, on the one hand, granted a long-awaited autonomy for regional universities, but wasted the opportunity to establish a modern state university system.
\end{abstract}


Keywords: University of Chile; University Reform; Campus Valparaiso; Counter Reform University; University of Valparaíso.

\section{Introducción}

La reforma universitaria en la entonces provincia de Valparaíso tiene como su principal protagonista a la Universidad Católica de Valparaíso y los sucesos acecidos en junio de 1967, que han sido considerados como el comienzo de dicho proceso, no sólo en la ciudad, sino también en el país, por la influencia posterior que tuvo en su similar santiaguina, la Pontificia Universidad Católica de Chile.

Producto de lo anterior, lo sucedido con la Universidad de Chile en Valparaíso ha pasado en gran medida desapercibido no sólo para el país, sino también en la región, siendo un aspecto desconocido incluso para los propios integrantes de la actual comunidad universitaria de la Universidad de Valparaíso, que no participaron en dicho proceso y que no han podido conocer con suficiente detalle los acontecimientos de entonces.

También ha influido en esta situación que lo sucedido en Valparaíso no siguió el mismo proceso de las otras sedes universitarias creadas en el país por la Universidad de Chile, donde la aparición paulatina de entidades de corte universitario como lo fueron los Colegios Regionales Universitarios $y$, posteriormente, los Centros Universitarios de Provincia durante la década de los sesenta, constituyó una gran novedad seguida y analizada con mucha atención, convirtiéndose en un paso previo para las respectivas sedes de la Casa de Bello. En cambio, lo sucedido en Valparaíso fue la aparición directa de la Sede Universitaria, en un lapso de tres años, en base a las escuelas e institutos ya instalados hacía varias décadas.

En consecuencia, el presente trabajo busca establecer los datos más relevantes para describir y caracterizar el surgimiento de la Sede Valparaíso de la Universidad de Chile, en la espera de la realización de un trabajo mucho más detallado y contundente sobre el particular, que considero indispensable para la mayor figuración de su sucesora, la Universidad de Valparaíso, más allá del ambiente universitario nacional, esto es, en la cultural general acerca de las universidades del país. 


\section{Constitución de la sede Valparaíso 1}

\subsection{Situación previa a la reforma}

Como pudimos exponer en un anterior trabajo, sobre el período previo al que analizamos, la existencia de la Universidad de Chile en Valparaíso era de tipo fragmentaria y dependiente de las autoridades universitarias con asiento en Santiago.2

Los primeros intentos de ordenamiento estaban orientados a un esfuerzo de tipo espacial-urbanístico, esto es, agrupar la mayor cantidad de escuelas e institutos universitarios creados en un solo lugar o sector de la ciudad, lo cual fue logrado sólo parcialmente. En dicha oportunidad hablamos de una "existencia inorgánica" y de la situación como "la Universidad de Chile en Valparaíso".3

Sin embargo, el problema no sólo era de tipo administrativo. El mundo y la universidad estaban cambiando, y las estructuras creadas hacía treinta años atrás ya no eran compatibles con la situación del momento. Se estimaba que había que ampliar el ámbito de acción de la universidad, que ésta no debía cerrarse sólo en la función de titular profesionales, sino también fomentar la investigación y abrir a las instituciones a la sociedad a través de la extensión y otras acciones complementarias. Las conversaciones dentro de la Universidad de Chile en torno a cómo encarar esta tarea se venían desarrollando desde hacía años, pero sólo a fines de la década de los sesenta el asunto tomó la trascendencia y urgencia necesaria para adoptar medidas concretas.

La Universidad de Chile en Valparaíso también estaba inmersa en ese proceso de grandes transformaciones, pero, en su caso, el camino a recorrer exigía un paso previo, consistente en el reordenamiento de todas las entidades existentes en la provincia de Valparaíso, a través de la creación de una estructura común y descentralizada de la autoridad santiaguina.

En esto también era diferente a las demás entidades provinciales de la Casa de Bello, puesto que éstas eran de reciente aparición, incipiente desarrollo y cada una organizadas bajo una sola autoridad. En cambio, las escuelas e institutos

\footnotetext{
1 Seguimos en gran parte lo expuesto en la obra de Carlos Martínez Corbella, única conocida que ha tratado el tema con gran extensión. También es recomendable revisar los Anales de la Universidad de Chile (julio-septiembre de 1968) que publica mucha documentación sobre el particular. Por su importancia para la historia de la Universidad de Valparaíso, es un tema que merece ser investigado con mayor detalle.

2 El texto se llama "Presencia de la Universidad de Chile en Valparaíso promediando el siglo XX (1940-1960). Antecedentes de la Universidad de Valparaíso" y permanece inédito.

3 Seguimos el esquema esbozado por el extinto profesor de la Escuela de Derecho de la Universidad de Valparaíso, Horacio Navarro Mayorga, quien distinguía tres períodos: Inorgánico (1911-1968); Sede Valparaíso (1968-1981); y Universidad de Valparaíso (1981-actualidad).
} 
porteños habían surgido hacía varios años, contaban con gran experiencia en su actividad académica, pero dependiendo cada una de la respectiva autoridad central santiaguina, restándoles actuación conjunta dentro del contexto de la provincia de Valparaíso.

Según relata Martínez Corbella, el asunto ya era discutido de forma abierta y con ideas estructuradas. Así, entre los años 1965 y 1967 rondaba la idea en la comunidad universitaria de "crear una Sede Autónoma de la Universidad de Chile en Valparaíso y sus alrededores, Viña y Montemar" (Martínez, 2002: 18). Según Pedrals, había cierto consenso entre las autoridades centrales y los directores de las escuelas porteñas "acerca de la creación de una Sede Autónoma de la Universidad de Chile en Valparaíso" (Pedrals, 2004: 85).

El mismo autor señala que, en 1966, mediante un Decreto Universitario se designó "una Comisión destinada a estudiar una mejor utilización de las Escuelas y Servicios Universitarios existentes en Valparaíso". Presidió esta comisión Héctor Echeverry e indica que "sus informes se constituyeron en valioso antecedente para la posterior creación de la Facultad de Valparaíso en 1968". Señala que a esta comisión se le denominó "Comisión de los Veintiuno" (Pedrals et al., 1982: 45). Como veremos, más adelante, según Martínez, esta "Comisión de los Veintiuno" es la presidida por Ventura Galván en 1967, un año más tarde. Es probable que haya existido otro grupo de personas que haya estudiado el tema con anterioridad a 1967 ya que, como mencionamos previamente, la idea venía discutiéndose años antes.

De lo transcrito, entendemos que se buscaba establecer una entidad descentralizada dentro de la Casa de Bello, y no de una universidad autónoma distinta de ésta, como se desprende en una primera lectura. Sin perjuicio de lo mencionado, hubo ciertos atisbos de establecer una entidad diferente de la Universidad de Chile. De esta manera se percibió, por ejemplo, respecto de una delegación de la ADIEX de Valparaíso (entendemos ADIV) que concurrió a una reunión en la capital, con una posición francamente separatista de la Universidad. Lo mismo se dijo de un acuerdo entre el Rector de la Universidad y la FECH, en que reconocerían, llegado el caso, la posibilidad de una "Universidad independiente" en Valparaíso (Anales, 1968: 136, 137 y 157).

\subsection{Constitución de la Sede Valparaíso}

Producto de esta situación, en 1967 el rector de la Universidad, Eugenio González Rojas, por Decreto de Rectoría № 1.867 conformó la denominada "Comisión Reestructuradora de la Universidad de Chile en Valparaíso", integrada por un representante del rector, seis directores de organismos universitarios de Valparaíso y otras personas que completaron un total de 21 miembros, razón por la 
cual fue conocida como "La Comisión de los 21". En representación del rector, se designó al decano de la Facultad de Arquitectura Ventura Galván Llorente, quién asumió la presidencia de la entidad. Posteriormente, se unieron a las conversaciones tres miembros del directorio de la Asociación de Docentes e Investigadores de Valparaíso (ADIV), quedando compuesta en total 24 miembros (Pedrals et al., 1982: 46).

Esta comisión entregó su informe al Rector en las primeras semanas de junio de 1968, con dos principales documentos: un anteproyecto de estatutos de la Sede Valparaíso y el anteproyecto de la estructura provisoria de la misma. Como para crear la Sede se necesitaba de la anuencia del Consejo Universitario, se sometió esta propuesta a este organismo colegiado, el cual en votación rechazó la iniciativa. A esta circunstancia se sumó el conflicto suscitado en la Facultad de Filosofía y Educación, que determinó la renuncia del rector González y su equipo, quien había hecho de esta reforma su principal bandera de lucha. Asumió como rector interino el decano de la Facultad de Agronomía, Ruy Barbosa Popolizio.

Mientras tanto, en Valparaíso el rechazo del proyecto y la renuncia del rector impulsor de la iniciativa fueron interpretados como un golpe fatal a las intenciones de descentralización. Para seguir impulsando la Reforma de la Universidad de Chile en Valparaíso y como medida de emergencia para no desistir y salvar todo lo avanzado, el 19 de mayo se constituyó la "Comisión Unida de Reforma", conformada por las tres agrupaciones gremiales de la universidad en la ciudad.

En primer lugar estaba la Asociación de Docentes e Investigadores de Valparaíso (ADIV) constituida en septiembre de 1967. Después se encontraba la Asociación de Profesores y Empleados de la Universidad de Chile-Valparaíso (APEUCH-V), que se había creado aproximadamente hacía doce años, tomando sólo relevancia en Valparaíso desde 1967 producto del proceso de reforma universitaria. Tiempo antes, se había escindido parte de la APEUCH, creándose la ADIEX (Asociación del Personal Docente, de Investigación y de Extensión) que existió exclusivamente en Santiago, con lo cual aquella entidad sólo quedó representando a los empleados (Anales, 1968: 169).4

Por último, encontramos a la Federación de Estudiantes de la Universidad de Chile-Valparaíso (FECH-V), que era la más antigua de las tres entidades. Fue fundada en abril de 1912, por los alumnos del entonces Curso Fiscal de Leyes de Valparaíso como asamblea provincial de la Federación de Estudiantes de Chile (FECH), pasando a llamarse con posterioridad Federación de Estudiantes de Valparaíso (FEV). A la fecha de la reforma, congregaba no sólo a los alumnos de la Escuela de Derecho de

4 Universidad de Chile-Sede Valparaíso. “Universidad de Chile-Valparaíso 1969-1970". Edeval. Valparaíso, 1971. pp. 69 y 71. 
Valparaíso de la Universidad de Chile, sino también a los estudiantes de las demás carreras de la Casa de Bello en la zona (Vergara, 2016: t. I. 336-338; t. II. 776).

Esta entidad triestamental convocó a una "Asamblea de la Comunidad Universitaria de Valparaíso" para forzar la creación, de todas maneras, de una Sede de la Universidad de Chile en Valparaíso.

En consecuencia, el 15 de junio de 1968, la asamblea de la comunidad reunida en el Aula Magna de la Escuela de Derecho de Valparaíso constituyó de facto y simbólicamente la "Sede Valparaíso de la Universidad de Chile", firmándose la respectiva acta, redactada por el asesor jurídico de la ADIV, el abogado y profesor de la Escuela de Derecho, Antonio Pedrals García de Cortázar.

Debe destacarse que dentro de la institucionalidad de la Universidad de Chile existente en ese momento, era imposible la creación de una "Sede", categoría que no existía en el estatuto universitario, de jerarquía legal, por lo cual había de utilizarse la estructura que estaba establecida, hasta la realización de una reforma mayor. 5 En consecuencia, -en palabras de Martínez Corbella- se propuso utilizar la estratagema de crear "la Facultad de Valparaíso", que era lo legal, para darle forma jurídica a la entidad. Tampoco podía hablarse de Rector en una sede, ya que ésta era la denominación para la jefatura superior nacional de la universidad, radicada en Santiago. En todo caso, le hubiera correspondido la denominación de "Vicerrector", la cual tampoco en los hechos podía utilizarse, debido a que no existía en el estatuto para ser utilizada en este caso (Martínez, 2002: 43). Como señala Pedrals, la solución en un principio pareció absurda, pero después fue utilizada llegándose a la paradoja que dentro de una Facultad se contenía, prácticamente, a una universidad completa. También, debe señalarse que no hay constancia cierta de la autoría de la solución que dio origen a la estructura de la naciente "Sede" Valparaíso (Pedrals, 2004: 85 y 86).

Una vez constituida de facto la Sede, se convocó a la "Primera Convención Regional de la Reforma", desde el 27 de junio al 9 de julio, la cual iba a discutir y preparar todos los proyectos relativos a estructura y funcionamiento de la nueva sede. En la primera sesión, se acordó sustituir la "Comisión Unida de Reforma", que había dirigido lo obrado desde el 15 de junio, por la "Comisión Regional de Reforma", que comenzó a funcionar el 11 de julio.

Esta nueva entidad iba a dirigir a la nueva sede en forma provisoria $y$, además, preocuparse de estructurarla para que comenzara a funcionar desde los primeros días de 1969. Para ello, una vez entregado el documento por dicha instancia, se convocó a referéndum para el 16 de agosto, en el cual se aprobó la propuesta general para redactar las normas que darían sustento jurídico efectivo a la

${ }_{5}^{5}$ Decreto con Fuerza de Ley № 280, publicado en el Diario Oficial de 30 de mayo de 1931. 
nueva Facultad-Sede. Así quedó abierto el proceso a la fase creación completa de la Sede a través de los mecanismos internos existentes, con la forma jurídica de Facultad de Valparaíso, lo que sucedió el 11 de octubre. En todo caso, siempre de pensó que esta labor era provisoria hasta que el Estatuto de la Universidad de Chile contemplara oficialmente la estructura de Sede.

Por otra parte, se abrió el proceso eleccionario para decidir quiénes iban ser el Decano y el Secretario de Facultad-Sede. Se fijó la elección para el 13 de septiembre y la asunción del mando en sus cargos para los elegidos, el 11 de octubre.

Habiéndose constituido de facto la Sede Valparaíso como Facultad el 15 de junio, se necesitaba dotarla de una estructura provisional, en espera del término del proceso que daría lugar a la estructura definitiva.

\section{Creación de la sede Valparaíso}

\subsection{Creación oficial y transitoria de la Sede Valparaíso}

Siendo esta una necesidad impulsada por la situación acaecida en la fecha ya mencionada, se procedió a establecer oficialmente dicha estructura. Por el Decreto Supremo № 8.309, del 11 de octubre de 1968, se creó la Facultad de Valparaíso con ámbito de acción en el territorio de la entonces provincia de Valparaíso, y reuniendo a todos los establecimientos de la Universidad de Chile radicados en ella, los que dejaban de depender de las Facultades de Santiago. Cabe recordar que a esta "Facultad-Sede" se le denominaba coloquialmente "Sede", aunque dicho nivel jerárquico no existía aun oficialmente dentro de la Universidad de Chile.

La estructura oficial y, a la vez transitoria, de la Facultad-Sede de Valparaíso, se estableció mediante el Decreto Supremo № 11.615 del 21 diciembre del mismo año. En ella se establecieron las llamadas "Áreas", las que asumirían las funciones de las tradicionales facultades, denominación que no podían usar debido a que ésta era utilizada para estructurar a la entidad porteña de la Casa de Bello.

Los servicios de las entidades que se detallan, eran traspasados a las nuevas "Áreas-Facultades", quedando de la siguiente forma:

a).- Área de Humanidades: Las actividades que se desarrollan en el Centro de Investigaciones de Historia Colonial y en el Instituto Pedagógico a excepción de las ejercidas en las Secciones de Matemáticas, Física, Química y Biología.

b).- Área de Ciencias Sociales: Las actividades que se desarrollan en las Escuelas de Economía, Servicio Social, y Ciencias Políticas y Administrativas (especialidad aduanera). La Escuela de Derecho de Valparaíso y el Instituto de 
Investigaciones Documentales, dependiente de esa Escuela, desarrollarán sus funciones estrechamente relacionadas con esta Área.

c).- Área de Arte y Tecnología: Las actividades que se desarrollan en la Escuela de Arquitectura y Centro de Investigaciones de Valparaíso.

d).- Área de Ciencias de la Salud: Las actividades que se desarrollan en las Escuelas de Odontología y Obstetricia. La Escuela de Medicina de Valparaíso desarrollará sus funciones estrechamente relacionadas con esta Área.

e).- Área de Matemáticas y Ciencias Naturales: Las actividades que se desarrollan en los Institutos del Departamento de Ciencias, Estación de Biología Marina, y en las Secciones de Matemáticas, Física, Química y Biología del Instituto Pedagógico (Anales, 1968: 299). 6

Ambas normas toman como sustento la letra d) del artículo 13 del Estatuto Orgánico Universitario de la Universidad de Chile que señala: "Corresponde al Consejo Universitario: d) Proponer al Ministerio de Educación Pública la creación, reorganización o supresión de Facultades, Escuelas e Institutos, Servicios y Establecimientos de la Universidad. No se necesitará la aprobación del Ministerio cuando se trate de Institutos que se costeen con los fondos propios de la Universidad".

Con base en esta disposición, se pudo estructurar provisionalmente la Facultad-Sede, mediante decretos, hasta la próxima reforma legal del Estatuto, que crearía la categoría de Sede, reconfigurando la organización de la Universidad de Chile.

La máxima autoridad "Facultad-Sede", era el cargo de decano, puesto para el cual fue elegido el arquitecto profesor Juan Araya Villarroel, siendo el secretario de la Facultad, el también arquitecto profesor Carlos Martínez Corbella.7 En todo caso debemos recordar que también, coloquialmente, a ambos cargos se les denominaba respectivamente "Vicerrector" y "Secretario General", aunque oficial y definitivamente eso sólo sucedió en mayo de 1972.

En jerarquía inferior quedaron las denominadas "Áreas-Facultad", que funcionaban prácticamente como una Facultad, a cargo de un "Director de Área". Esta estructura reemplazó, dentro de la nueva organización de la actividad universitaria, a las antiguas Escuelas, y reunió dentro de sí a los respectivos Departamentos, encabezados por los "Directores de Departamentos".

6 Se dejó fuera a las Escuelas de Derecho y de Medicina por tener ambas una ley que las favorecía de forma directa. La Escuela de Enfermería, hasta ese momento dependiente del Hospital Carlos van Buren, sería integrada a la Sede Valparaíso en 1970.

7 La elección se efectuó el viernes 13 de septiembre de 1968, y al no haber candidatos que obtuvieran mayoría absoluta, se procedió a una segunda votación el viernes 4 de octubre siguiente. La asunción en los cargos se hizo el viernes 11 de octubre del mismo año en el Aula Magna de la Escuela de Derecho. 
En cuanto a las oficinas para la nueva jefatura superior de la recién constituida Facultad-Sede, la llamada coloquialmente "Vicerrectoría", se ocupó un sector del edificio de la Escuela de Derecho, el ubicado en la calle Blanco con General Cruz, denominada comúnmente "la punta de crucero". Debe recordarse que esta construcción, concluida definitivamente en 1957, en los hechos se convirtió en la edificación icónica no sólo de dicha escuela universitaria, sino también de la Universidad de Chile en Valparaíso.8

La nueva institucionalidad comenzó a funcionar al año siguiente con los recursos existentes hasta ese momento, ya que su instalación no conllevaba la entrega de nuevos fondos, por lo cual se encontraba con escaso financiamiento.

\subsection{Estructura Académica de la Sede}

La constitución de la Facultad-Sede no sólo fue la ocasión de reordenar a las escuelas e institutos de la Universidad de Chile en Valparaíso bajo una autoridad común provincial dentro de la universidad, sino también sirvió para otorgarle un nuevo enfoque, desde el punto de vista de la administración académica.

En efecto, en el proyecto se estableció como unidad básica el "Departamento" en lugar del tradicional concepto de "Escuela", que había dominado desde sus inicios a las instituciones creadas en la ciudad, sin perjuicio de la aparición de los Institutos como entidades científicas. Por otra parte, el concepto de carrera ya no se relacionaba únicamente con una Escuela y con la enseñanza de una profesión, sino que se concebía como "un curriculum que se cumple en los diversos Departamentos de la Sede y que habilita para obtención de un título profesional".

Dentro de esta nueva estructura, el Departamento se encargaba de las actividades de investigación, docencia y extensión de una determinada rama del saber, en la cual tenía su campo de acción de forma exclusiva, evitando duplicación de recursos y aprovechándolos al máximo. A este proceso se le denominó "departamentalización".

A continuación, surgiría el Área (Facultad) cuya principal función sería coordinar e integrar la investigación, docencia y extensión de todos los departamentos que lo componían, tomando el lugar antes ocupado por las Escuelas e Institutos. Por otra parte, también propendería a centralizar racionalmente la administración y servicios técnicos de los departamentos y carreras profesionales.

\footnotetext{
8 Sin perjuicio del edificio de la Estación de Biología Marina de Montemar y el perteneciente al Instituto Pedagógico, hoy Casa Central del Universidad de Playa Ancha. El edificio de la Escuela de Derecho es hasta la actualidad representativo de la Universidad de Valparaíso, mientras no se construya una Casa Central.
} 
Por último, la Sede como organismo regional que tenía por objeto cumplir con los objetivos de la Universidad de Chile en las provincias de Valparaíso y Aconcagua (Badenier, 2017: 23).9

Todo lo anterior ya había sido discutido antes de los hechos precedentemente descritos, por lo cual los acontecimientos posteriores tuvieron por objeto ratificar lo ya acordado y ponerlo en práctica en la nueva Sede.

De acuerdo a la nueva estructuración, las modificaciones de rigor y realizada la planificación detallada de todos los aspectos necesarios, las carreras según las respectivas Áreas a las cuales estaban adscritas eran las siguientes:

1).- Área de Arte y Tecnología: arquitectura, cinematografía, construcción civil, dibujo técnico, diseño industrial y teatro.

2).- Área de Ciencias Sociales: administración aduanera, contadores auditores, derecho, ingeniería comercial, periodismo y servicio social.

3).- Área de Ciencias de la Salud: enfermería, medicina, obstetricia y odontología.

4).- Área de Humanidades: pedagogías en alemán; biología y ciencias; castellano, educación física; educación general básica; francés; filosofía; historia, geografía y educación cívica; inglés; matemáticas y física; química y ciencias; consejeros educacionales y vocacionales; y bibliotecología.

5).- Área de Matemáticas y Ciencias Naturales: licenciatura en matemática y biología marina. 10

\subsection{Consejo Coordinador Universitario de Valparaíso}

Una consecuencia insospechada de la constitución de la Sede Valparaíso, fue la creación en 1969 del denominado "Consejo Coordinador Universitario de Valparaíso", una instancia que reunía a la naciente Sede Valparaíso de la Universidad de Chile, a la Universidad Católica de Valparaíso y la Universidad Técnica Federico Santa María. Su objetivo era hacer causa común en la obtención de fondos para el desarrollo de las universidades en la entonces provincia. Según lo relatado por Martínez Corbella en su obra, la idea habría surgido en una reunión sostenida por él y Juan Araya Villarroel en busca de soluciones a la falta de recursos para la naciente Sede de la Casa de Bello, lo que se logró con el acuerdo de los rectores de las otras universidades, creando una entidad que permitiera hacer causa común frente a la

9 UniversidAd DE ChILE-Sede VAlParaíso. "Universidad de Chile-Valparaíso 1969-1970". Edeval. Valparaíso, 1971. pp. 17-20.

10 UniversidAd DE ChILE-Sede VAlParaíso. "Universidad de Chile-Valparaíso 1969-1970". Edeval. Valparaíso, 1971. pp. 44, 46, 50, 53 y 58. El detalle de los programas de las carreras, ver en Universidad de Chile, Sede de ValParaíso. Oficina de Planificación. Planes de Estudio. Planificación Curricular 1970. Edeval, 1970. 
autoridad central. Raúl Allard entrega un dato interesante: el acuerdo de instalación de este Consejo se habría suscrito el 10 de septiembre de 1969, siendo los rectores Jaime Chiang Acosta por la UTFSM, Raúl Allard Neumann por la UCV, y Juan Araya Villarroel por la Sede Valparaíso de la Universidad de Chile. Para su funcionamiento se creó el cargo de Secretario Ejecutivo y se dictó una ley especial para su financiamiento (Martínez, 2002: 49-50, 239, 247; Allard, 2002: 133-137).11

Esta entidad es el origen del actual Consejo de Rectores de Valparaíso (CRUV), que es integrada por las universidades mencionadas y, también, por la Universidad de Valparaíso y la Universidad de Playa Ancha, como sucesoras de la antigua Sede Valparaíso de la Universidad de Chile.12

\subsection{Creación definitiva de la Sede Valparaíso}

Entretanto se establecían las normas definitivas de la reforma en Valparaíso, el Decreto Supremo № 1.186, de 6 de marzo de 1970, modificó el texto del Decreto № 4.859 reemplazando la expresión "Centros Universitarios", por "Sedes Universitarias" (López et al., 1980: 9). Recordemos que las unidades universitarias de Valparaíso se constituyeron en una Facultad para llegar organizarse en una Sede; mientras que las demás entidades de la Universidad de Chile en el país surgieron desde los centros universitarios de provincia habiendo sido algunos previamente colegios universitarios regionales. En ambos casos, se producía un paso intermedio en la reforma, a diferencia de Valparaíso.

El largo proceso de reformar la Casa de Bello se realizó a través de la "Comisión Nacional de la Reforma de la Universidad de Chile", cuya labor terminó en 1971 con la publicación del nuevo Estatuto Orgánico Universitario que reemplazaba en gran parte al antiguo de 1931.13 En su artículo 11, definía a las Sedes oficialmente como parte de la estructura universitaria; y, en su artículo primero transitorio, mencionaba a las existentes hasta ese momento: Arica, Iquique, Antofagasta, La Serena, Valparaíso, Talca, Ñuble, Temuco y Osorno, que correspondían, salvo en el caso de Valparaíso, a los antiguos Centros Universitarios de Provincia y, en otros tantos, a los otrora Colegios Regionales Universitarios. Se menciona a Santiago también como una Sede, con la posibilidad de dividirla si fuere

\footnotetext{
11 Ley № 17.394, publicada en el Diario Oficial de 26 de diciembre de 1970, cuyo artículo 6o consideraba a la Facultad de Valparaíso de la Universidad de Chile al mismo nivel de las otras universidades, y a su decano como su representante legal. Si su denominación o estructura variaba, seguiría recibiendo los aportes. Dicha norma fue dejada sin efecto durante el gobierno militar.

12 Sitio Web DEl ConSEjo de ReCTORES DE VALPARAíso http://consejoderectoresvalparaiso.cl/ (consultado el 27.10.2017). La norma que lo rige es el Decreto № 571, publicado en el Diario Oficial de 13 de diciembre de 1978.

${ }_{13}$ Decreto con Fuerza de Ley № 1, publicado en el Diario Oficial de 8 de junio de 1971.
} 
necesario, lo cual sucedió más tarde al crearse las Sedes Santiago Norte, Sur, Oriente y Occidente.

En cuanto a nuestro objeto de análisis, el paso definitivo se dio con el Decreto Universitario № 4.513, de 29 de mayo de 1972, en que se creó oficial y estructuralmente con el nombre correspondiente la "Sede Valparaíso".

Su composición era la siguiente (Pedrals et al., 1982: 50):

1).- Facultad de Salud.

2).- Facultad de Arte y Tecnología.

3).- Facultad de Ciencias Sociales.

4).- Facultad de Educación y Letras (Ex Humanidades).

5).- Facultad de Matemáticas y Ciencias Naturales.

Ahora, la Sede Valparaíso, que por convencionalismo se había llamado así, aunque estructuralmente era una Facultad, tenía una autoridad máxima con nombre nuevo: Vicerrector. En el fondo como "Sede" sus funciones seguían siendo las mismas y las "Áreas", ahora Facultades, también. Siguió en dicho cargo, ahora con el nuevo nombre oficial, el arquitecto profesor Juan Araya Villarroel, hasta el término de su mandato en 1972, siendo reemplazado como vicerrector por el hasta ese momento Secretario General de la sede, profesor arquitecto Carlos Martínez Corbella. Por su parte, se eligió como nuevo secretario general al Profesor de Estado Jorge Baldrich Álvarez (Martínez, 2002: 60).14

Todo este proceso iniciado en junio de 1968, fijado de forma provisional en octubre y diciembre de ese mismo año, consolidado en junio de 1971 con el nuevo estatuto orgánico de la Universidad de Chile y con la creación oficial de la Sede Valparaíso en mayo de 1972, terminaría primero de facto y, posteriormente en forma jurídica, con el advenimiento del gobierno militar.

\section{Intervención militar}

Con el 11 de septiembre de 1973, la reforma es parcialmente desmantelada. En efecto, la estructura descentralizada de sedes, facultades y departamentos continuó vigente, con algunas modificaciones menores. Sin embargo, la forma de elección de las autoridades y selección de su profesorado fue suprimida, sin perjuicio de los despidos y otras situaciones más dramáticas que se dieron en aquella época.

En el caso de la Sede Valparaíso, su vicerrector salió al exilio. En primera instancia se nombró, en calidad de Rector-Delegado de la Universidad de Chile en Valparaíso, a Andrés Barros Pérez-Cotapos, odontólogo y profesor de la Escuela del

14 Efectuada el jueves 28 de septiembre de 1972 y asumiendo el cargo el miércoles 11 de octubre del mismo año, por el periodo 1972-1976, que quedó inconcluso por la llegada del gobierno militar. 
ramo en la sede, quien era además Capitán de Fragata de la Armada, Sanidad Naval. Como Vicerrector de la Sede al doctor Pedro Uribe Concha, a la fecha Decano de la Facultad de Salud y, como Secretario General, al abogado y profesor de la Escuela de Derecho, Guillermo Schiessler Quezada.

De estos nombramientos llama la atención la función asumida por Pedro Uribe Concha. Aunque se le designó como Vicerrector de la Sede, estaba actuando en conjunto con el Rector-Delegado de ésta, con una evidente colisión de funciones. Las notas periodísticas posteriores, lo mencionaron como "Vicerrector Académico", denominación más específica y que no producía choque de cometidos entre ambas autoridades.

Otro asunto de importancia consiste en una situación que ha pasado desapercibida hasta la actualidad. Al poco tiempo de asumir el Rector-Delegado, se proyectó descentralizar la Sede Valparaíso de la Universidad de Chile, que presentaba una dependencia de los aspectos docentes y administrativos de la Casa Central en Santiago, pasando a llamarse "Universidad de Chile de Valparaíso", una sede independiente de la Universidad de Chile, conservando los vínculos con ésta, en especial, en cuanto a programación y planificación.

Esta es la primera ocasión en que se intentó separar a la Sede Valparaíso de la Universidad de Chile, pero sin perder los vínculos con su matriz, entendemos en un paso más avanzado de lo existente hasta ese momento. Hacemos notar que en reportajes posteriores se persistió en la denominación "Universidad de Chile de Valparaíso", según estas ideas descentralizadoras.

De acuerdo a Badenier, el doctor Barros Pérez-Cotapos, a quien se refiere como "delegado-militar" y no como Rector-Delegado de la Sede, "fue un fuerte partidario de la autonomía de la sede en contraposición a lo que sostenía el rector Delegado de la Universidad de Chile; General Agustín Toro Dávila, cuestión que a Barros le costó el cargo" (Badenier, 2017: 56).15

En consecuencia, este intento tuvo poca duración. Más adelante se designó para el cargo de Vicerrector de la Sede al General de Ejército (R) Ramón Salinas Figueroa, quien ejerció el puesto entre 1974-1981, último año en el cual se creará la Universidad de Valparaíso. Esto implicó reencauzar el manejo de la Sede de acuerdo a las normas ya existentes, con lo cual este breve intento fue producto de una situación circunstancial y no parte de un proyecto de mayor alcance. 16

\footnotetext{
${ }_{15}$ Agustín Toro Dávila no era el rector de la Universidad de Chile en dicho período, sino el General del Aire César Ruiz Danyau. Sitio Web Universidad DE ChILE, en específico, http://www.uchile.cl/portal/presentacion/historia/rectores-de-la-u-de-chile/4720/periodo-derectores-delegados-1973-1990 (consultado 19.6.2019).

16 El Mercurio de Valparaíso. 23 de septiembre de 1973. Primer Cuerpo, páginas 2 y 3; 27 de septiembre de 1973. Primer Cuerpo, página 5; 11 de octubre de 1973. Primer Cuerpo, página 9; 21 de diciembre de 1973. Primer Cuerpo, página 2.
} 
Una víctima impensada de los acontecimientos, fue la aspiración de trasladar parte de la Sede Valparaíso al parque Sausalito, previo convenio con la Ilustre Municipalidad de Viña del Mar, existiendo un proyecto de construir en dicho lugar las instalaciones de la Facultad de Arte y Tecnología (habiendo planos y dibujos). Después de lo acaecido, el proyecto fue desahuciado por las nuevas autoridades (Martínez, 2002: 23, 59, 60, 185, 189 y 191).

Con la Reforma Administrativa iniciada en 1974, las sedes provinciales de la Universidad de Chile serán "Sedes Regionales", y continuarán su labor con el mismo estatus hasta la Reforma Universitaria de 1981, que desmembrará a la Universidad de Chile de sus sedes regionales.

\section{Creación de la Universidad de Valparaíso}

\subsection{La Contrarreforma Universitaria del Gobierno Militar}

Dentro de las reformas que el Gobierno Militar quería implantar en el país según su ideario, estaba una intervención profunda en el sistema educacional y las universidades existentes en ese entonces.

En consecuencia, en diciembre de 1980 la Junta de Gobierno dictó el Decreto Ley № 3.541, por el cual se facultó al Presidente de la República a reestructurar las universidades del país, incluida la Universidad de Chile, "pudiendo dictar todas las disposiciones que fueren necesarias al efecto $y$, en especial, aquellas destinadas a fijar su régimen jurídico y a regular el establecimiento de corporaciones de esta naturaleza, pudiendo en el ejercicio de estas atribuciones, dictar normas estatuarias o de procedimientos para regular su estructura orgánica". Remataba la ley señalando que las facultades se ejercerían por medio de decretos con fuerza de ley. Por su parte, el diario El Mercurio de Valparaíso, señalaba que la reforma se originaba en la Directiva Presidencial de Educación, de marzo de 1979, por la cual se había creado una comisión para estudiar las reformas al sistema universitario.17

En virtud de esta norma, se dictaron varios Decretos con Fuerza de Ley: DFL № 3, de 31 de diciembre de 1980, sobre remuneraciones en universidades chilenas; DFL № 1, de 3 de enero de 1981, que fija normas sobre universidades; DFL № 2, de 7 de enero de 1981, fija normas sobre universidades; y DFL № 4, de 20 de enero de 1981, que fija normas sobre financiamiento de universidades. Más adelante, se dictaron los DFL № 5, de 16 de febrero de 1981, que fija normas sobre institutos profesionales; y el DFL № 24, de 16 de abril de 1981, que fija normas sobre centros

17 Promulgado el 12 de diciembre de 1980 y publicado en el Diario Oficial al día siguiente; EL MercuRio de VAlParaíso. 13 de diciembre de 1980. Primer Cuerpo, páginas 1 y 12. 
de formación técnica. ${ }^{18}$ Todas estas normas conformaron el proceso de reforma universitaria impulsado por el Gobierno Militar, que sus detractores han denominado "contrarreforma universitaria", por desmantelar el sistema universitario existente hasta el momento, en todos sus aspectos.

De todas estas normas, la directamente relacionada con el proceso sufrido por la Sede Valparaíso de la Universidad de Chile, fue el DFL № 2, que fijaba normas sobre Universidades, facultando a los rectores de las universidades existentes para proponer al Presidente de la República un programa de reestructuración de las respectivas entidades. Se establecía la posibilidad de crear nuevas universidades, adoptándose las medidas del caso. ${ }^{19}$

El entonces ministro del Interior, Sergio Fernández, explicó los objetivos del decreto ley, entre otros, elevar la calidad de la vida universitaria y racionalizar las actuales universidades, "particularmente las que en mayor medida se han visto afectadas por un crecimiento inorgánico y desmesurado". Más adelante, señala que la reestructuración implicaba "cuanto procediere, incluso en un plan de división para alguna de las actuales universidades, de modo que se conviertan en dos o más entidades enteramente autónomas y diferentes". En todo el caso, el proceso no afectaría a los actuales alumnos ni el otorgamiento de los títulos. 20

\subsection{Fin de la Sede Valparaíso y regresión transitoria}

Producto de este afán reformador, manifestado particularmente en el DFL № 2, la Sede Valparaíso de la Universidad de Chile experimentó una situación muy poco conocida en la actualidad, consistente en que durante el lapso de un mes aproximadamente, prácticamente desapareció de la estructura institucional de la Universidad de Chile.

Una primera señal de lo que vendría surgió a mediados de enero. El Mercurio de Valparaíso de 17 de dicho mes, señaló que estaba finalizando la etapa de reestructuración de la Universidad de Chile. En esencia, las autoridades centrales y la cobertura nacional se mantendrían, pero se efectuarían ciertas modificaciones, como la supresión de ciertas sedes regionales, manteniendo aquellas que cumplieran con los fines universitarios. En general, otro objetivo era racionalizar el sistema aquejado por sobreoferta de carreras, abundancia de programas y un desequilibrio entre la oferta académica y las oportunidades de trabajo según las carreras. 21

\footnotetext{
18 Las fechas indicadas se refieren a su publicación en el Diario Oficial. Los DFL № 1 y 2 poseen el mismo nombre oficial.

19 Promulgado el 30 de diciembre de 1980 y publicado en el Diario Oficial de 7 de enero de 1981.

20 El MercuRio de Valparaíso. 8 de enero de 1981. Primer Cuerpo, páginas 1 y 10.

21 El MercuRio de Valparaíso. 17 de enero de 1981. Primer Cuerpo, páginas 1 y 12.
} 
Sin embargo, el gran golpe se daría unos días más tarde. Así, el citado diario con fecha 21 de enero publicaba en su portada "Suprimen vicerrectoría y decanatos en la U. de Chile". La nota periodística desarrollaba las principales medidas a adoptar: supresión de las vicerrectorías en las sedes regionales, reducción de las facultades santiaguinas de diecinueve a diez, las que tomarían el control de las desaparecidas facultades de las sedes, la coordinación de éstas con el nivel central sería a través del Secretario Universitario Regional. Las medidas no afectarían a las carreras y los alumnos, pero tendría algunos efectos en el personal, los cuales procurarían ser los necesarios en aras de la racionalización. El objetivo era lograr una mayor descentralización académica, agilizando el quehacer universitario.22

En resumen, se eliminaba de la estructura de la Sede Valparaíso su vicerrectoría y cinco facultades, pasando a depender los departamentos y las carreras de las respectivas facultades santiaguinas, ahora con cobertura nacional. Esta nueva situación implicaba suprimir la esencia de la sede sin eliminarla formalmente, constituyendo la sentencia de muerte para todo lo que se había estructurado preparatoriamente desde mediados de los años sesenta, constituido en 1968 y consolidado en 1972. El centralismo, con nuevos ropajes, volvía en gloria y majestad a la Universidad de Chile.

Como es de suponer, la noticia caló hondo en la universidad. El Mercurio de Valparaíso tituló en portada "Caso U. de Chile: 'grave atentado a Valparaíso"'. La nota periodística explicó que lo esperado de la reforma era reforzar la autonomía de la Sede Valparaíso y no someterla a dependencia de Santiago. Esta decisión implicaba la dejación del cargo del vicerrector Ramón Salinas Figueroa, y de los cinco decanos de facultad: Italo Paolinelli Monti (Ciencias Jurídicas, Económicas y Sociales), Carlos Patillo Bergen (Facultad de Medicina), Hugo Moletto Sola (Arte y Tecnología), Olga Arellano Salgado (Educación y Letras), y Tatiana Alviña Walker (Matemáticas y Ciencias Naturales). El vicerrector haría entrega del cargo al secretario general, Sergio de Tezanos Pinto, el 22 de enero, a las 11:00 horas. Esta autoridad quedaría en funciones durante la fase de reestructuración, en cumplimiento de las nuevas disposiciones. ${ }^{23}$

Los comentarios no se dejaron esperar. El Secretario General, de Tezanos Pinto, hizo una breve descripción al tomar su cargo. Señaló que prácticamente se había enterado de sorpresa de la decisión y que muchas cosas eran inciertas aún. Las medidas adoptadas retrotraían la situación al año 1967, yendo en contra de todos los logros: primero la Facultad, después la Vicerrectoría, e incluso un proceso que logró disminuir la burocracia que había crecido mucho logrando dejar sólo los

\footnotetext{
22 El MerCuRio de Valparaíso. 21 de enero de 1981. Primer Cuerpo, páginas 1 y 11.
}

23 El Mercurio de Valparaíso. 22 de enero de 1981. Primer Cuerpo, páginas 1 y 7. 
servicios generales en Vicerrectoría. Estimaba que no habría mucha reducción de personal, debido a que el proceso se había dado paulatinamente con el retiro o jubilación de varios funcionarios, por lo cual se funcionaba con el personal indispensable. Lo que quedaba era adaptarse a la situación y planificar el inicio de actividades. 24

También hizo sus comentarios el renunciado vicerrector, Ramón Salinas Figueroa. Señaló que las medidas adoptadas lo habían tomado por sorpresa, ya que lo esperado era una mayor independencia para la sede. De hecho, señaló que su mayor aspiración era lograr independizar plenamente a la Sede Valparaíso como una universidad propia, siendo la tercera de la región, quedando a la par de las demás, especialmente para efectos de las reuniones del Consejo Regional de Rectores, que no podía adoptar decisiones por su cargo de vicerrector. De hecho, Valparaíso era prácticamente una universidad y necesitaban la autoridad de un rector propio, lo que había conversado con el exrector Toro Dávila. La posibilidad también se habría hablado con el Presidente de la República, pero fue rechazada por motivos económicos. Detalló después su plan para reunir las diversas carreras dispersas en ciertos lugares específicos de Valparaíso y Viña, eliminar algunas de estas que estaban sobredimensionadas, y reabrir la carrera de periodismo, con énfasis en la especialización. Además, habló sobre otros proyectos de adelanto para el personal de la institución y algunas unidades académicas. 25

Asimismo, manifestaron sus impresiones tres de los ex decanos de las desaparecidas facultades de Ciencias Jurídicas, Económicas y Sociales, Italo Paolinelli Monti; de Educación y Letras, Olga Arellano Salgado; y de Medicina, Carlos Patillo Bergen. En general, las tres autoridades expusieron que la decisión los había tomado por total sorpresa, que era algo que nadie había esperado, que tampoco se les expuso motivo alguno y que la decisión afectaba a la región y a la ciudad. Además, señalaron que se pensaba de alguna u otra forma en la creación de una universidad autónoma con su propio rector, aunque en el caso de Paolinelli tenía sus reservas respecto de mantener el prestigio y nombre independientes de la Universidad de Chile. También se pensaba que era algo que atentaba contra la idea de la regionalización y que se le infería un gran daño a la ciudad. En todo caso, señalaban que había de esperarse los resultados que darían las nuevas medidas. ${ }^{2} 6$

Por su parte, los profesores de la ex Facultad de Medicina de Sede, enviaron una carta al rector de la Universidad, Alejandro Medina Lois, en la cual, aceptando la situación y ofreciendo su máxima colaboración, se cuestionaban el porqué de la

24 El Mercurio de Valparaíso. 22 de enero de 1981. Primer Cuerpo, páginas 1 y 7.

25 El Mercurio de ValParaíso. 23 de enero de 1981. Primer Cuerpo, páginas 1 y 7. El discurso de despedida del Vicerrector, mismo diario, 24 de enero de 1981. Primer Cuerpo, página 4.

26 El Mercurio de Valparaíso. 25 de enero de 1981. Primer Cuerpo, página 7. 
decisión adoptada, máxime cuando la restructuración en vez de otorgar más autonomía, los hacía depender de la Facultad de Medicina en Santiago, contrariando la política de descentralización impulsada por el CONARA (Uribe, 1985: 145-147).27

Por último, los decanos de Santiago efectuaron una visita a Valparaíso, con el objeto de coordinar la toma de control de las entidades porteñas. Se estableció que el secretario general seguiría en sus funciones hasta la designación de la nueva autoridad, denominada Secretario Universitario Regional. Las adecuaciones que había de hacerse eran de tipo administrativo y académico, sin afectar mayormente a los funcionarios y alumnos. Se volvió a señalar que la idea era racionalizar el funcionamiento de la entidad.28

Lo único que puede comentarse al respecto, más allá de lo reseñado, es la reiterativa invocación del argumento de la racionalización de funcionamiento de la entidad, que fue aplicada a tal extremo que no puede evitarse pensar que era totalmente contraproducente, trayendo más burocracia y complicaciones. La medida era totalmente errada, puesto que lo mejor era utilizar las estructuras existentes de las sedes y aprovechar la experiencia haciendo algo más ordenado, que volver a centralizar todo.

Como nada se les dijo a las autoridades en su momento, hasta donde sabemos, probablemente nunca se sabrán las razones de fondo de tan desafortunada medida.

\subsection{Creación de la Universidad de Valparaíso}

Este era el ambiente cuando el día 6 de febrero, el diario El Mercurio de Valparaíso publica la noticia de la creación de la "Universidad de Chile de Valparaíso". Se señala que esta decisión se habría adoptado en vista de la reacción causada en la ciudad ante la decisión de la reestructuración total del plantel que había sido anunciada el 20 de enero anterior, con los trastornos provocados en la administración interna, el golpe a la frustrada idea de una universidad autónoma y por las gestiones del vicealmirante Raúl López Silva, intendente regional. La nota también transcribe la entrevista al abogado Renato Damilano Bonfante, quien fue designado por el Presidente de la República como Rector de la Universidad.29

Con fecha 7 de febrero, El Mercurio de Valparaíso, publica una serie de breves entrevistas a varias personalidades de la ciudad en torno a la creación de la nueva universidad. Todas ellas destacan la instalación de la nueva institución de educación

27 Comisión Nacional de la Reforma Administrativa, creada por el DL № 212, de 26 de diciembre de 1973, con el objeto de estudiar e implementar una reforma a la Administración del Estado.

28 El MercuRio de Valparaíso. 28 de enero de 1981. Primer Cuerpo, página 3.

29 El MercuRio de VAlPARAíso. 6 de febrero de 1981. Primer Cuerpo, páginas 1 y 3. 
superior, aseverando que era un gran avance en la descentralización, destacando la personalidad de su rector, que se dio marcha atrás en la tendencia recentralizadora que observaba desde Santiago, expresan los desafíos para ponerla en marcha en tan poco tiempo, y la vinculación con la actividad marítima que se le auguraba. Particular mención debemos hacer de dos aspectos: en primer lugar, que su creación fue decisión directa del Presidente de la República y, de hecho, fue la primera de todas las nuevas universidades estatales en ese período. También resalta lo aseverado por el rector Damilano respecto de su nombre: la entidad era autónoma, totalmente desligada de la Universidad de Chile, por lo cual "Incluso nuestro propio nombre no debe recordar en nada a la Universidad de Chile, por eso lleva solamente el apellido de Valparaíso". 30 Hasta poco tiempo antes, se hablaba de la "Universidad de Chile de Valparaíso", tal como sucedió en octubre de 1973, pero desde este momento en adelante, se denominaba a la institución oficialmente como la "Universidad de Valparaíso".

Siguiendo en el tiempo, el 8 de febrero el mismo diario anunció que el martes siguiente se crearía la Universidad. También realizó una entrevista al ex vicerrector Ramón Salinas, que mostró su beneplácito por la creación de la nueva entidad, viendo que se cumplía una de sus más grandes aspiraciones y por la cual había luchado mucho. Justifica su denominación oficial, excluyendo a la Universidad de Chile, porque debe tener su personalidad propia ligada a la ciudad. Manifiesta su entusiasmo por el desarrollo que le proyecta, en especial su tinte regional y marítimo, y espera que pueda seguir expendiéndose a otros sectores como el interior de la región. También se entrevistó al ex decano de Arte y Tecnología, Hugo Moletto Sola, quien manifestó que la ex Sede Valparaíso había logrado su mayoría de edad en lo formal y académico, por lo cual merecía ser reconocida como Universidad y que, en los hechos, funcionaba como tal. También destacó que las facultades no habían sido un regalo, sino que se habían ganado con esfuerzo en todos los ámbitos del quehacer universitario. Terminó señalando que debía haber un apoyo desde la Universidad a la comunidad y viceversa. Por su parte, la editorial del diario hizo una observación importante: el nuevo rector era el primer civil en dicho cargo, tomando en cuenta que sus pares eran todos militares, situación existente desde 1973.31

Con fecha 10 de febrero, el mismo diario entrevista al Secretario General de la Sede, aún en funciones, Sergio de Tezanos Pinto, quien relata todos los pasos dados para organizar la nueva universidad y cómo se va a efectuar el proceso de postulación a ésta, para los alumnos que iniciarán sus estudios en la nueva universidad. Anuncia la ceremonia de inauguración de la nueva entidad, a realizarse

3o El MERCURIo de ValParaíso. 7 de febrero de 1981. Primer Cuerpo, páginas 1 y 3.

31 El Mercurio de Valparaíso. 8 de febrero de 1981. Primer Cuerpo, páginas1 y 12. La editorial del diario sobre el particular, página 5. 
el 10 de febrero, a las 11:30 horas, en el Aula Magna de la Escuela de Derecho, con la presencia del Presidente de la República, quien firmará el decreto de creación de la institución. 32 En esa misma edición, la editorial del diario hacía un recuento histórico a grandes rasgos del contexto en que surge la nueva institución, dentro del cual destacamos el siguiente párrafo: "Así pues, como queda explicado, la Universidad que hoy nace no es una Universidad nueva que debe dar a tientas sus primeros pasos. Es una Universidad que luce con orgullo una antigua tradición, un largo historial de eficaces servicios en la educación y en la investigación, y un ancho y bien cimentado prestigio". 33

Finalmente, el mismo diario publicó las noticias sobre la ceremonia de creación de la Universidad, realizada el día 10 de febrero. En ella participaron el Presidente de la República y autoridades nacionales y regionales. Hizo uso de la palabra el rector de la Universidad de Chile, general Alejandro Medina Lois, el intendente de Valparaíso, vicealmirante Raúl López Silva, y el Ministro de Educación, Alfredo Prieto, quien leyó una carta de agradecimiento al ex vicerrector Ramón Salinas. En ese mismo acto, se procedió a designar como rector a Renato Damilano Bonfante. La nota periodística señaló que el nuevo rector, procedió a nombrar como prorrector a Sergio de Tezanos Pinto, ex secretario general de la Sede, pidiéndoles también la colaboración a los ex decanos Italo Paolinelli, Carlos Patillo y Hugo Moletto, con el objeto de hacer funcionar la nueva institución. Señala que aún faltaban los estatutos y dictar toda la normativa interna de la institución. Destaca que la naciente universidad era la novena casa de estudios universitarios de Chile.

Por su parte, ese mismo día, la nota editorial comentaba los sucesos, recordando el origen de la nueva universidad hacía setenta años atrás, con la creación de la carrera de Derecho en 1911. Asimismo, destaca un hecho relevante: es la primera vez desde aquella época, que los estudios universitarios de la institución estatal en Valparaíso recibían el estatus legal que siempre había merecido. Remarca también que la nueva universidad era la novena en el país. Por último, señala: "Hito fundamental de la historia de Valparaíso ha sido la fundación de la Universidad de su nombre, en el día de ayer". ${ }^{34}$ Solo queda comentar algo al respecto: al ser de origen estatal, la Universidad no fue fundada, sino más bien "creada", tanto porque así lo dice el respectivo texto legal que le da existencia como porque la expresión "fundación" es más apropiada para actos de una persona o grupo de personas particulares, que para un acto de una autoridad estatal.

\footnotetext{
32 El MERCURio de ValPaRAíso. 10 de febrero de 1981. Primer Cuerpo, páginas 1 y 12.

33 El MercuRio de ValParaíso. 10 de febrero de 1981. Primer Cuerpo, página 5.

34 El Mercurio de Valparaíso. 11 de febrero de 1981. Primer Cuerpo, páginas 1 y 7. Para ver las cartas al lector y la editorial, páginas 4 y 5 .
} 
Por su parte, Badenier entrega una mirada desde el punto de vista de los académicos y funcionarios. Dice que, por una parte, esta medida seguía la estrategia del gobierno de "cercenar a la Universidad de Chile como universidad de carácter nacional, de modo de establecer un mayor control político de la ya menguada actividad académica existente en país". Pero también observa que este proceso "daba satisfacción a aquellos que pensaban que la plena autonomía de la Universidad en Valparaíso era la base indispensable para su crecimiento y desarrollo ante las eternas barreras burocráticas de la casa central en Santiago" (Badenier, 2017; 89).

La Sede de la Vicerrectoría en el sector de la calle General Cruz se convirtió, desde entonces, en la sede de la rectoría de la nueva universidad, permaneciendo allí durante varios años. Posteriormente se instaló en su lugar la Contraloría Interna de la institución hasta 2015, cuando el recinto fue entregado a la Facultad de Derecho.

\subsection{Repercusiones}

Entonces, con la creación de la Universidad de Valparaíso, por el DFL № 6, publicado en el Diario Oficial de 12 de febrero de 1981, estructurada en base a la Sede Valparaíso de la Universidad de Chile, se convirtió en la continuadora para todos los efectos legales, la entidad ya señalada. ${ }^{5}$ Cabe hacer notar que esta norma hablaba todavía de la "Sede Valparaíso" que existía formalmente, aunque su estructura había sido eliminada a mediados de enero del mismo año. También, mediante el Decreto Supremo № 500, de 12 de febrero de 1981 se designó como Rector de la nueva universidad al profesor de la Escuela de Derecho, Renato Damilano Bonfante. 36

Más adelante, con fecha 11 de diciembre de 1981 se dictaría el DFL № 147, Estatuto de la Universidad de Valparaíso, otorgándole estructura propia y definitiva. ${ }^{7}$ Posteriormente, se dictó el Reglamento Orgánico de la Universidad de Valparaíso, contenido en el Decreto Universitario № 480, de 26 de octubre de 1983. Con ambas normas, se retornó a la estructura clásica de Facultades como, asimismo, de Escuelas e Institutos, dando por terminado el sistema de departamentalización establecido por la Reforma Universitaria de 1968.38

\footnotetext{
35 Promulgado el 10 de febrero de 1981.

36 Publicado en el Diario Oficial de 26 de febrero de 1981.

37 Publicado en el Diario Oficial de 2 de abril de 1982.

38 La estructura interna era la tradicional y básica, semejante a un servicio público, quedando desfasada con el transcurso del tiempo, por lo que se requería una modernización, dentro del marco que aún rige. En noviembre de 2012, se aprobó mediante referéndum, el proyecto de nuevo Estatuto Orgánico para la Universidad de Valparaíso, al cual le falta su sanción como DFL de parte del Presidente de la República. Por otra parte, el 15 de marzo de 2017, mediante Decreto Exento №
} 
En consecuencia, la creación de la Universidad de Valparaíso era algo notable, ya que de todas las sedes de la Universidad de Chile, aquélla era la que poseía las escuelas e institutos más antiguos y funcionando muchos años antes de la creación de todas las sedes regionales, por lo tanto tenía la mayor experiencia y prestigio acumulado desde el punto de vista académico y administrativo, siendo la mejor candidata a independizarse.

También se cumplía con un viejo anhelo, surgido casi al mismo tiempo de la creación del antiguo Curso Fiscal de Leyes en 1911, consistente en asegurar la existencia de las entidades universitarias en Valparaíso con una norma de jerarquía legal, y no sólo con una de carácter reglamentario, mucho más expuesta a ser suprimida que aquélla (Vergara, 2016: t. I. 364-365; Maluenda, 1947: 7).

Si se observa con atención, casi todas las escuelas e institutos creados en Valparaíso aparecieron por decreto y, más adelante, también la misma Facultad-Sede provisoria de 1968, como la Sede definitiva de 1972 (aunque estaba mencionada en el estatuto universitario de 1971). Es decir, todo el sistema se estructuraba con una normativa fácilmente derogable, lo que no sucedería con una consagración legal que asegurara, al menos, la existencia de una universidad propia para la región. Con ello se evitaría que volviera a suceder lo acaecido entre enero e inicios de febrero de 1981, con la práctica desaparición de la antigua Sede Valparaíso de la Universidad de Chile.

Un claro ejemplo de lo anterior lo ofrece la historia de la Escuela de Derecho. En efecto, durante su existencia estuvo expuesta a ser cerrada en diferentes ocasiones en los años 1914, 1926, 1930, 1931 y 1942 e, incluso, se habló de su cierre a principios de 1981, siendo una institución suficientemente consolidada con casi setenta años de existencia. Esto reflejaba el irracional centralismo santiaguino y justificaba plenamente la creación vía legal de la universidad porteña (Vergara, 2016: t. I: 365-367, 496-501; t. II: 612-614; 636-638 y 712).

Sin perjuicio de lo anteriormente señalado, debemos hacer una observación interesante y que refleja la forma de funcionamiento de nuestra institucionalidad educacional: como ha quedado establecido, la primera vez que se habló claramente de crear una entidad estatal universitaria en la ciudad, fue una solicitud elevada por estudiantes del entonces Liceo de Valparaíso, en agosto de 1881, pidiendo la instalación de un curso de leyes pagado por el gobierno, teniendo como antecedente la entidad organizada bajo la rectoría de Eduardo de la Barra. Después de un extenso proceso se logra crear oficialmente en 1911 el Curso Fiscal de Leyes, que a la larga

1.253, se aprobó el nuevo Reglamento Orgánico de la Universidad de Valparaíso. Las nuevas autoridades superiores asumieron sus cargos a inicios de 2018. EL MERCURIO DE VALPARAíso. 6 de enero de 2018. Página 8. SITIO WEB UniversidAD DE VALPARAíso, específicamente http://www.uv.cl/pdn/?id=9304 (consultado 7.1.2018). 
fue la inspiración para la creación de diversas escuelas e institutos, o bien, de intentos de instalar una universidad estatal nueva, tras lo cual transcurren casi exactamente cien años hasta febrero de 1981, con la creación de la Universidad de Valparaíso. Desde aquella lejana fecha, tomó prácticamente un siglo consolidar firmemente una institucionalidad universitaria estatal en Valparaíso (Vergara, 2016: t. I. 153-154).

Lo anteriormente expuesto debe hacernos reflexionar cómo se manejan los tiempos y las prioridades en nuestro país, respecto de dotar a las regiones de entidades educacionales de parte del Estado. Da mucho que pensar el que la solicitud original se haya hecho en la denominada "época de gloria" de la ciudad y la consolidación de la universidad estatal se produjera en tiempos de franca decadencia de ésta, en un proceso con muchos sobresaltos y retrocesos.

Si bien es cierto este aspecto merece un estudio y análisis más profundo y completo, no deja de llamar la atención el notorio desfase entre la necesidad invocada y su realización efectiva. De esto sólo hacemos mención, principalmente, para extraer las lecciones de dicha circunstancia.

\subsection{Consecuencia imprevista}

Sin embargo, el proceso de autonomía para la nueva universidad derivó en una fragmentación de un importante componente. A principios de marzo de 1981, el gobierno militar dictó el DFL № 13, que creó el Instituto Profesional denominado "Academia Superior de Ciencias Pedagógicas de Valparaíso", estructurado en base a la Facultad de Educación y Letras de la recién creada Universidad de Valparaíso. 39 Mediante el Decreto № 1.316, de 21 de marzo de 1981, se designó como su rectora a doña Olga Arellano Salgado.40 Más adelante, mediante la ley № 18.434, de septiembre de 1985, se convertirá en la actual Universidad de Playa Ancha.41

Esto significó un doble golpe para la novel universidad: en primer lugar, se le separó de un importante miembro constituido por el antiguo Instituto Pedagógico de Valparaíso, quedando excluida de la formación de los futuros profesionales de la educación. Por otra parte, al establecerlo como "Instituto Profesional", regulado por el reciente DFL № 5, le rebajó desde el nivel universitario a otro inferior, en contra de todo lo que indicaba la tradición y lo recomendado por la experiencia.42

El desmembramiento de las sedes regionales de la Universidad de Chile y la Universidad Técnica del Estado, su fusión para crear las universidades estatales

\footnotetext{
39 Promulgado el 10 de marzo de 1981, y publicado en el Diario Oficial de 20 de marzo siguiente.

40 Publicado en el Diario Oficial de 11 de abril de 1981.

41 Publicada en el Diario Oficial de 4 de septiembre de 1985.

42 Promulgado el 6 de febrero de 1981, y publicado en el Diario Oficial de 16 del mismo mes.
} 
regionales, más la nueva estructuración totalmente opuesta a la consagrada por la reforma de los años sesenta, constituyen la denominada "contrarreforma universitaria", que dio origen, en gran medida, al actual sistema universitario. Fuera de las universidades tradicionales y sus derivadas, surgirán, además, las primeras universidades privadas. 43

Por otra parte, no deja de llamar la atención el errático proceder del gobierno de turno sobre el particular. Contando con comisiones de estudio y pleno poder, inició una reforma con efectos totalmente contraproducentes, dando marcha atrás al poco tiempo, decidiendo crear universidades estatales nuevas en pleno proceso de admisión de estudiantes a la educación superior, con todos los trastornos del caso, y generando incertidumbre en los funcionarios, demostrando que las autoridades no estaban muy convencidas de las decisiones adoptadas.

En ese sentido, este proceso fue muy desprolijo, contrastando con lo acaecido en la creación de las nuevas universidades estatales de O’Higgins y Aysén en 2016, cuyo surgimiento tuvo un itinerario mucho más lento y participativo, sin librarse de diversas críticas. Aunque son dos épocas diferentes en muchos aspectos, no hay similitud entre ambos casos, salvo que en el primero no había oportunidad alguna de oponerse de parte de las entidades y, en el segundo, hubo todo un debate y planificación respecto de su instalación y funcionamiento, sin librarse del todo de críticas, en especial, de sus similares del sector privado de más reciente creación.

\section{Conclusiones}

Como se puede apreciar en este trabajo, la creación y funcionamiento de la Sede Valparaíso de la Universidad de Chile fue una experiencia con consecuencias relevantes para la historia de las universidades en la actual región de Valparaíso.

En efecto, su aparición respondió a las aspiraciones que venían prácticamente generándose con fuerza desde principios del siglo XX, en orden que el Estado diera cobertura a las necesidades de la educación superior en la ciudad, ya fuera a través de una universidad distinta a la Casa de Bello, o bien, en una sede de ésta.

El surgimiento de la Sede Valparaíso, reuniendo bajo una autoridad común a las entidades porteñas de la Universidad de Chile, fue un acontecimiento que tomó tiempo siendo resistido por muchas instancias internas a nivel central en la Casa de Bello, hasta que la comunidad universitaria en la ciudad logró forzar su creación. Esta resistencia, en la gran mayoría de los casos, fue constante durante el surgimiento de las diversas unidades académicas de la Casa de Bello en la ciudad. Lamentablemente, los primeros años de la Sede Valparaíso transcurrieron en una de

43 La Universidad Gabriela Mistral en 1981; la Universidad Diego Portales en 1982; y la Universidad Central en 1983. El resto de las universidades privadas surgirá desde fines de dicha década. 
las épocas más difíciles de la historia nacional, inmersa en un grave conflicto político, ideológico e institucional.

Producto de la Reforma Universitaria su creación fue una evolución importante en el desarrollo de la Universidad de Chile en la entonces provincia, reuniendo a todos sus integrantes bajo un autoridad común y propia, significando la modernización de una institución con años de presencia en la ciudad. Sin embargo, en los hechos, el proceso no fue suficiente ya que la entidad operaba como una universidad, pero con cierto nivel de dependencia de la administración central, dejándola en clara desventaja frente a sus pares porteñas. En consecuencia, el proceso constituyó el primer paso para la creación de una nueva universidad para la ciudad y la región, lo que le otorga a la Reforma de la Universidad de Chile en Valparaíso un carácter distintivo frente a lo sucedido con sus congéneres.

Posteriormente, un proceso de reforma claramente centralista y retrógrado, determinó en un primer momento que las autoridades de la época decidieran finalmente una verdadera supresión de la Sede Valparaíso para, posteriormente, invertir dicha decisión y proceder a la creación de la Universidad de Valparaíso y, consecuencialmente, de la Universidad de Playa Ancha.

Tal como antes, la presión de la ciudadanía ante equivocadas decisiones a nivel central, logró la creación de una universidad estatal independiente, plenamente autónoma de la Universidad de Chile. El intento de reestructuración, fue el último acto de las numerosas ocasiones en que las actitudes centralizadoras de la Universidad de Chile respecto de sus unidades académicas en Valparaíso. Sólo es posible imaginar qué destino hubiera tenido la Casa de Bello, de haberse descentralizado paulatinamente, reconociendo gradualmente más autonomía a sus sedes regionales. También es una notable paradoja de la historia, que su creación se hubiera efectuado durante un gobierno de facto.

La creación de la nueva universidad constituyó un gran avance para la región, pero se efectuó dentro del contexto de una reforma universitaria que dañó severamente a las Universidades del Estado en su conjunto, cuyos efectos se padecen hasta el día de hoy y tratan de corregirse de la mejor forma posible.

Este aspecto es quizá uno de los más particulares del proceso: por una parte, la creación de la Universidad de Valparaíso fue un triunfo por sobre las tendencias centralistas que buscaban resucitar una Universidad de Chile centralizada, desperdiciando toda la experiencia ganada durante los trece años de existencia de la sede porteña. En cierto modo, fue la consecución de una aspiración que había sido acariciada durante muchos años. Sin embargo, dentro del contexto de la reforma impulsada por el Gobierno Militar, significó la desintegración de la Universidad de Chile, en vez de establecer un sistema de universidades estatales autónomas, robusto y cohesionado, al servicio del país. Fue un evento de dulce y agraz. 
El presente trabajo no sólo es un recordatorio de la historia reciente de las universidades estatales en la zona, sino también pretende servir de guía para la mejor comprensión y proyección de la educación superior estatal en la región de Valparaíso. En particular, es un aliciente para que la Universidad de Valparaíso a través de futuras investigaciones asuma la labor impostergable y esencial de recuperar los antecedentes históricos de esta época relevante en su creación y primera existencia.

\section{Bibliografía}

Allard, R. (2002): “35 Años Después. Visión Retrospectiva de la Reforma 1967-1973 en la Universidad Católica de Valparaíso". Ediciones Universitarias de Valparaíso. Valparaíso.

Badenier, O. (2017): "Entre Sonrisas, Alegrías y Desencantos". Universidad de Valparaíso-Chile. Facultad de Odontología. Valparaíso.

López, I.; D. Véliz y M. Gamonal (c. 1980): "Historia de la Sede Arica de la Universidad de Chile 1960-1980". Arica.

Maluenda, L. (1947): “Apuntes y Notas para una Historia de la Escuela de Derecho de Valparaíso". Memoria de prueba para optar al grado de licenciado en la Facultad de Ciencias Jurídicas y Sociales de la Universidad de Chile. Valparaíso, Imprenta Londres.

Martínez, C. (2002): "La Sede Valparaíso de la Universidad de Chile. La Reforma Universitaria 1968-1973. Memorias de un académico". Puntángeles Universidad de Playa Ancha Editorial. Valparaíso.

Pedrals, A.; H. Navarro y A. Topasio (1982): "La Escuela de Derecho de la Universidad de Valparaíso". Valparaíso, EDEVAL.

Pedrals, A. (2004): "El sueño de Eduardo de la Barra", en Memoria. Valparaíso, EDEVAL.

Uribe, P. (1985): "La Escuela de Medicina de Valparaíso". Valparaíso. Imprenta Victoria. 
Vergara, M. (2016): "El Curso Fiscal de Leyes de Valparaíso. Desde los Cursos Libres de Leyes hasta la Escuela de Ciencias Jurídicas y Sociales de Valparaíso (1878-1934). La primera época de la Escuela de Derecho de la Universidad de Valparaíso". Edición Privada. Dos Tomos.

Fecha de recepción: 4 de diciembre de 2019.

Fecha de aceptación: 7 de enero de 2010. 